\title{
Temporal interfaces and metamaterial response enabled by boundaries
}

\author{
Luca Stefanini, Davide Ramaccia ${ }^{\dagger}$, Alessandro Toscano and Filiberto Bilotti
}

“Roma Tre” University, Department of Engineering, Via Vito Volterra 62, 00146, Rome, Italy

(completed on 01 April 2021)

\begin{abstract}
In the last years, temporal metamaterials have been exploited as a novel platform for conceiving several electromagnetic and optical devices based on the anomalous scattering response achieved at a single or multiple sudden change of their medium properties, which however is difficult to carry out in a realistic scenario In this work we investigate on the possibility to emulate the scattering response of a temporal metamaterial without acting on the medium properties, but on the effective refractive index and wave impedance perceived by the wave during the propagation within a guiding structure rather than the actual material, by acting on its boundaries. The work presents in closed form the scattering coefficients achieved when the boundary properties are suddenly modified for inducing an effective temporal interface. In this framework, temporally controlled metasurfaces can be used to implement the proposed concept, giving an easier path also to the design and realization of novel devices at microwave and optical frequencies.
\end{abstract}

a) ${ }^{\dagger}$ To whom correspondence should be addressed: ${ }^{\dagger} \underline{\text { davide.ramaccia@uniroma3.it. }}$ 
Temporal metamaterials are artificial electromagnetic materials whose effective medium properties vary over time. In case of abrupt changes of medium properties, the propagating wave experiences a scattering process like the one experienced at the interface between two different media, realizing the temporal counterpart of the well-known spatial discontinuity of the medium properties. It is common to refer to this instant of time as temporal interface that is characterized by the generation of a refracted and a reflected wave, whose amplitudes and frequencies are related to the jump of the refractive index and wave impedance between the two media, i.e., before and after the changing [1,2]. It is clear that the possibility to control the scattering also using the time dimension have drawn significant attention in recent years [3,4]. Like in single or multilayered spatial structures, playing with the temporal sequence and application time of the materials over the propagation period enables plenty of intriguing wave phenomena [5], e.g., inverse prism [6], temporal aiming [7], and synthesis of effective media [8], as well as novel devices in time-domain, e.g. antireflection coatings [9,10], temporal Fabry-Perot cavities [10], spacetime crystals [11], and broadband absorbers [12]. However, these recent works focus on switching the electromagnetic and optical properties of infinite media, which is difficult to carry out in any realistic scenario, both for experimentally validate the envisioned phenomena and for the actual implementation of the proposed devices.

In this work, we raise the question as to whether it may be possible to act on the effective medium parameters, perceived by guided wave, instead of the actual media parameters, for realizing a temporal interface, and thus a temporal metamaterial. In this case, the temporal interface would be induced by just acting on the bounding surfaces of a waveguide, relaxing the modulation of the bulk media supporting the propagation. In Fig. 1, we illustrate the two possible strategies for enabling boundary-induced interfaces within a guiding structure: i) changing the properties of the surfaces bounding the waveguide, represented by the arbitrary surface impedance $Z$ (Fig. 1a-c); or ii) changing the distance between two surfaces with $Z=0$, i.e., conventional metallic plates (Fig. 1b-d). 
Bounday-induced Spatial interfaces
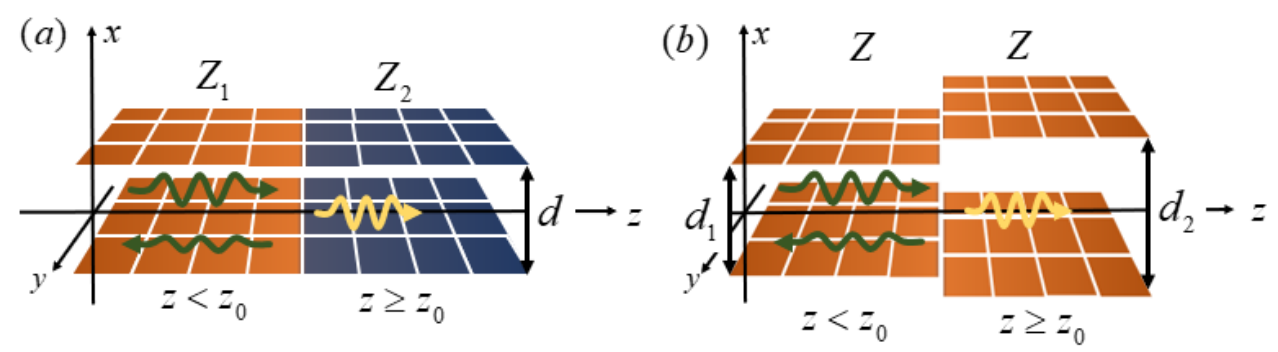

Bounday-induced Temporal interfaces
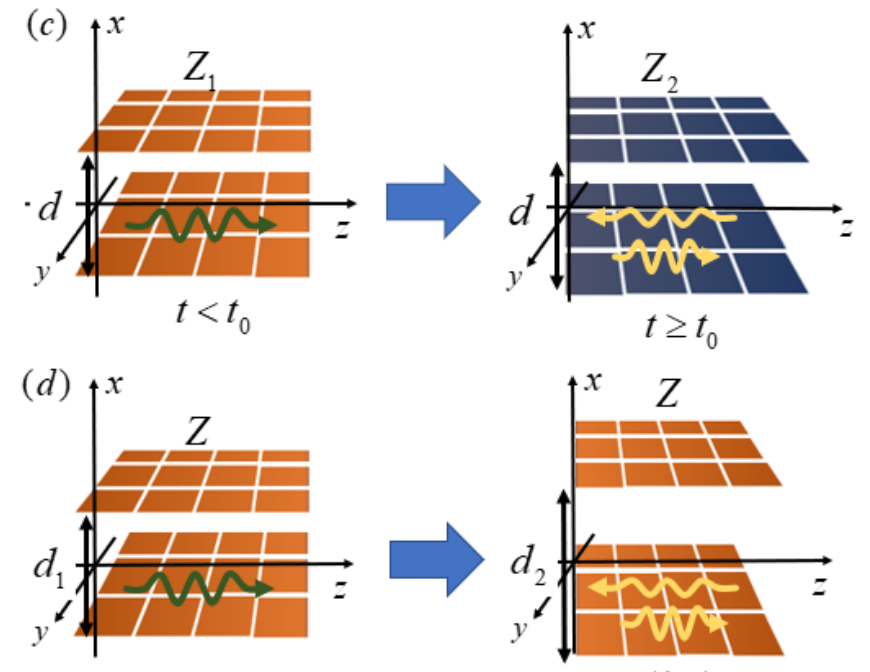

$t<t_{0}$

$t \geq t_{0}$

Fig. 1. Graphical illustrations of wave propagation discontinuities induced by boundaries in a guiding structure: (a) spatial and (c) temporal interfaces induced by a change of the bounding surface properties; (b) spatial and (d) temporal interfaces induced by a change of the waveguide dimensions.

The former represents an interesting strategy for actual implementation of the boundary-induced interfaces, thanks to possibility to leverage on the engineerable surface properties of the metasurfaces. For example, Fig. 1a shows the case of two waveguides bounded by different metasurfaces realizing a spatial interfaces, and Fig. 1c shows its temporal counterpart where the surface properties are modulated in time switching from $Z_{1}$ to $Z_{2}$ at $t=t_{0}$ [13-17]. The analysis of this configuration can be performed starting from the knowledge of the eigenmodes of a metasurface-bounded waveguide for a given pair of the metasurfaces, as derived recently by Ma et al. in [18]. However, the latter strategy, i.e., moving the metallic plates, better highlights the physical process triggered by an abrupt modification of the boundary conditions over time, allowing to catch all the interesting features of the proposed approach for inducing a temporal interface through the boundary conditions. Therefore, from now on, we narrow the premise to this second case, that 
does not affect the validity of the proof and corresponding results in general. The other case is sufficiently like the one investigated here that proving it follows by essentially the same logic.

Looking at the well-established waveguide theory [19], the connection of two different waveguides as shown in Fig. 1b leads inevitably to a scattering process at the interface, as shown in Fig. 1b. This can be related to the conservation of the transversal wave quantities, i.e., electric, and magnetic fields, since the two waveguides support two separate sets of eigenmodes of the propagating field and only generating a reflected and refracted wave allows matching them at the discontinuity location $z_{0}$. Fig. $1 \mathrm{~d}$ illustrates the same abrupt change of the waveguide dimensions but in time domain. Before the switching time $\mathrm{t} 0$, the propagating mode is defined by the excitation frequency $\omega_{1}=2 \pi f_{1}$, the waveguide dimension $\mathrm{d} 1$ and the electromagnetic properties of the filling medium. At the instant $\mathrm{t} 0$, the waveguide dimension changes to $\mathrm{d} 2$. In the following, we investigated it in the details, relating the electromagnetic response of the system to the change of the effective media, which is in general different from the actual media filling the waveguide, experienced by the wave at the temporal discontinuity interface [19].

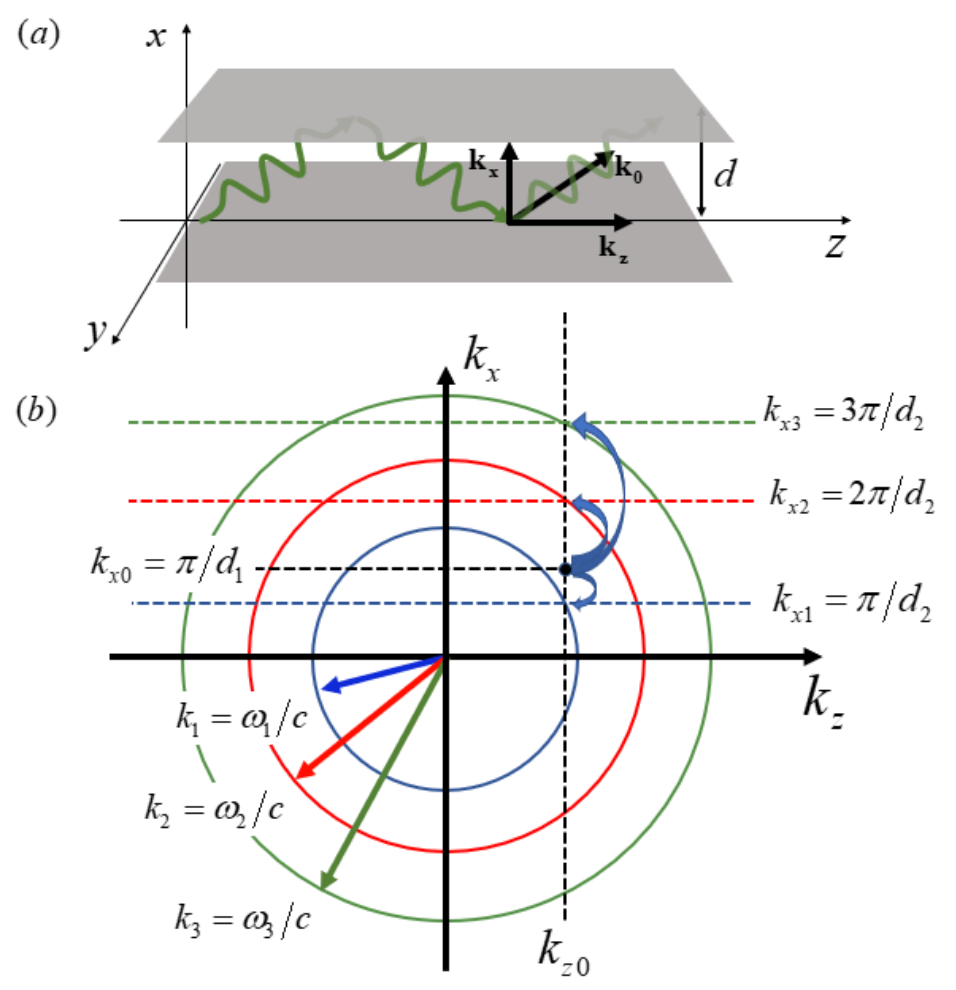

Fig. 2. (a) Representation of the propagation quantities of the TE/TM modes within a parallel-plate waveguide of dimension $\mathrm{d}$; (b) dispersion diagram of excited modes supported by the waveguide after a temporal discontinuity induced by a sudden change of the waveguide dimensions from $d=d_{1}$ to $d=d_{2}$. 
Let us consider an infinitely extended parallel-plate waveguide (PPWG) consisting of two metallic plates separated of a distance $\mathrm{d}$ in the $\mathrm{x}$-direction and supporting the propagation of a $\mathrm{z}$-directed wave, as shown in Fig. 2a. The waveguide is filled with vacuum $(n=1)$. The propagation of the fundamental TEM mode is always supported at any frequency, whereas the TE/TM modes are degenerate and supported after the corresponding cut-off frequencies, dictated by the electrical dimension $d / \lambda$ of the waveguide. In this scenario, it is worth remembering that the TEM mode always travels with the same phase velocity within the waveguide regardless the distance d, making it insensitive to the boundary-induced temporal discontinuity. This allows us neglecting it in our dissertation and focus on the TE/TM modes. In addition to that, TE/TM modes are degenerate, relaxing the analysis to only one of the set of modes. In the following, we consider a waveguide supporting the propagation of the first TM mode, but a similar discussion and results can be derived straightforwardly for the TE mode case. Fig. 2a shows the propagation of the TM1 mode within a PPWG. The propagation vector $\mathbf{k}_{z}$ is the projection of the filling medium wavevector $\mathbf{k}_{0}$, whose magnitude is $k_{z}=\sqrt{k_{0}^{2}-k_{x}^{2}}$, where $k_{0}=\omega / c=2 \pi / \lambda$ is the filling medium wavenumber, and $k_{x}=\pi / d$ is the transverse wavenumber imposed by the waveguide dimensions. The effective refractive index perceived by the guided mode is:

$$
n_{e f f}=\sqrt{1-\left(k_{x} / k_{0}\right)^{2}}=\sqrt{1-(\lambda / 2 d)^{2}}
$$

and its phase velocity is $v_{p}=\omega / k_{z}=\omega / \sqrt{k_{0}^{2}-(\pi / d)^{2}}$.

Figure $2 \mathrm{~b}$ represents the excited modes in the parallel plate waveguide in the dispersion diagram at the temporal interface (Fig.1d). Before the time ${ }^{t_{0}}$, the fundamental TM1 mode is propagating with a propagation wavenumber $k_{z 0}$ and a transverse wavenumber $k_{x 0}$, identified by dashed black lines in Fig. $2 \mathrm{~b}$. The distance between the origin of the axes and their point of intersection is the wavevector $\mathbf{k}_{0}$ (not represented in Fig.2b). At the temporal discontinuity, ${ }^{t=t_{0}}$, the original propagating mode TM1 perceived an instantaneous change of the effective medium from $n_{\text {eff }}\left(d_{1}\right)$ to $n_{\text {eff }}\left(d_{2}\right)$, which inevitably induces the instantaneous change of the temporal frequency though the scaling time-dilation factor $\xi_{[5,10] \text { : }}$ 


$$
\omega_{1}=\xi \omega_{0}, \text { where } \xi=\frac{n_{e f f}\left(d_{1}\right)}{n_{e f f}\left(d_{2}\right)}=\sqrt{\frac{1-\left(k_{x 0} / k_{0}\right)^{2}}{1-\left(k_{x 1} / k_{1}\right)^{2}}}
$$

and the generation of a reflected and refracted wave. It is worth remembering that the wavelength is conserved, and so also the propagating wavevector ${ }^{k_{z}}$. Therefore, not only the first TM1 mode in the new waveguide is excited, but also all the TM modes defined by the transverse wavenumbers $k_{x n}=n \pi / d_{2}$ with $n=1,2,3, \ldots$, and the same original propagation wavenumber $k_{z}=k_{z 0}$ [1]. In Fig. 2b, the circles crossing the points of intersection between $k_{z 0}$ and $k_{x n}$ represent the wavevectors ${ }^{k_{n}}$ of the new modes propagating in the waveguide, each of which propagates at a different frequency as $\omega_{n}=c k_{n}$. Therefore, for $t \geq t_{0}$, the original propagating wave at frequency $\omega_{0}=c k_{0}$ becomes evanescent being not supported yet, and its energy is distributed among the modes with $k_{z}=k_{z 0}$ and propagating at frequencies $\omega_{n}=c k_{n}$. In Figure $3 \mathrm{a}$ and $3 \mathrm{~b}$, we report the H-field map and the spectra of the incident and scattered fields computed numerically through a Finite Difference Time Domain (FDTD) simulation, respectively. At the instant of time ${ }^{t=t_{0}}$, the original PPWG with dimensions $d_{1}=\lambda_{0} / 15$ increases its dimensions to $d_{2}=1.2 d_{1}$ starting the leakage process of energy from the original mode to all supported modes of the new waveguide with the same propagating wavenumber. Thanks to the knowledge of $k_{z 0}$ and $k_{x n}$, we can easily derive (3) that allows evaluating analytically the new frequencies after the boundary-induced temporal interface:

$$
\omega_{n}=\omega_{0} \sqrt{1-\frac{k_{x 0}^{2}-k_{x n}^{2}}{k_{0}^{2}}}, \quad n=1,2,3, \ldots
$$

Figure $3 \mathrm{c}$ shows the comparison between the analytical (solid line) and numerical (crosses) output normalized frequencies for the first three modes excited in the new waveguide after the boundary induced temporal interface as a function of the relative jump in the dimensions of the waveguide $d_{2} / d_{1}$. These modes deliver almost all the energy of the original TM1 propagating in the waveguide before $t_{0}$ due to their lower 
phase mismatch between the original propagating and final supported modes. The agreement between numerical and analytical results is very good, confirming the process illustrated in Fig. $2 \mathrm{~b}$.

In addition to the excitation of the supported modes at different frequencies, as in any discontinuity the temporal interface generates always a backward (reflected) and forward (transmitted) wave, shown in Figure

3a for ${ }^{t>t_{0}}$. To evaluate in closed form the reflection and transmission coefficients at the boundary-induced temporal interface, we have to split the problem in two phases: first, we impose the continuity of the fields at the interface and derivates the instantaneous response of the guided wave to the change of the effective refractive index, and then, the coupled mode theory (CMT) is used for describing how the reflected and refracted TMn modes in the new waveguide are excited. This allows evaluating the corresponding transmission and reflection coefficients in closed form for the TM modes. The same equations can be used for the evaluation of the reflection and transmission coefficient in case of propagation of the TE mode. Imposing the continuity condition of the H-/E-field components between the initial fundamental TM1 mode and the final TM1 mode across the discontinuity we have:

$$
\begin{aligned}
& \left(H_{i}\right)_{t=t_{0}^{-}}=\left(H_{r}+H_{t}\right)_{t=t_{0}^{+}} \Rightarrow H_{0}^{y}=\left(A e^{i \omega_{1} t_{0}}+B e^{-i \omega_{1} t_{0}}\right) H_{1}^{y} \xi^{-1} \\
& \left(E_{i}\right)_{t=t_{0}^{-}}=\left(E_{r}+E_{t}\right)_{t=t_{0}^{+}} \Rightarrow E_{0}^{x, z}=\left(A e^{i \omega_{1} t_{0}}-B e^{-i \omega_{1} t_{0}}\right) E_{0}^{x, z}
\end{aligned}
$$

where A, B are the amplitudes of the forward (trasmitted) and backward (reflected) propagating waves respectively and is the time dilation factor due to the instantaneous frequency shift induced by the boundaries. Here, we simply imposed the continuity of H-/E-field in (4) because the filling medium of the waveguide does not change across the interface, relaxing the continuity of $\mathrm{B} / \mathrm{D}$ to $\mathrm{H} / \mathrm{E}$. However, after the discontinuity, the modes excited in the waveguides split the energy of the new fundamental mode according to their coupling coefficient. Equation (4) can be written considering this process, expanding the second term in the modes supported by the waveguide:

$$
\begin{aligned}
& \kappa_{0 n} H_{0}=\left(a_{n} e^{i \omega_{n} t_{0}}+b_{n} e^{-i \omega_{n} t_{0}}\right) H_{n} \xi^{-1} \\
& \kappa_{0 n} E_{0}=\left(a_{m} e^{i \omega_{n} t_{0}}-b_{m} e^{-i \omega_{n} t_{0}}\right) E_{n}
\end{aligned}
$$


where $\kappa_{0 n}$ is the coupling coefficient giving by the overlap integral on the initial cross section and $a_{n}, b_{n}$ are the amplitudes of the forward and backward propagating waves of each mode propagating within the PPWG, respectively. In (5), the coupling coefficient plays an important role in the selection of the excitable modes: despite the wavelength matching exists for every mode index $n$ with both even and odd symmetry, the energy distribution can suppress certain modes if the overlap integral is zero due to the opposite symmetry with respect to the waveguide axis. This motivates the presence of the only odd modes after the boundary induced temporal discontinuity.

Assuming that the amplitude of the original mode decreases exponentially as $\exp [-\alpha t]$, we can solve (5) and derive the scattering coefficients of all new excited modes within the waveguide:

$$
\left\{\begin{array}{l}
T_{H}=\frac{k_{x n}}{2 k_{x 0}}\left(1+\frac{\omega_{0}}{\omega_{n}}\right) \kappa_{0 n}\left(\frac{\omega_{0}}{\omega_{1}}\right) \\
R_{H}=\frac{k_{x n}}{2 k_{x 0}}\left(1-\frac{\omega_{0}}{\omega_{n}}\right) \kappa_{0 n}\left(\frac{\omega_{0}}{\omega_{1}}\right)
\end{array}\right.
$$

The reflection and transmission coefficients in (5) are reported in Fig3d and Fig. 3e, respectively, as a function of the jump between the initial and final waveguide. The analytical results are compared with the numerical ones showing a very good agreement between them.

To conclude, we have discussed the possibility to induce a temporal interface by acting on the effective medium properties rather than the actual bulk material supporting the propagation. This has been demonstrated though the relevant example of propagation within a parallel plate waveguide whose boundary conditions change suddenly. We have described in detail the physical process in the transformation from the initial to the final field configuration and we also derived analytically the frequency shifts imposed by the boundary-induced temporal interfaces and the corresponding reflection and transmission coefficients. The analytical results have been compared with FDTD numerical results showing a good agreement between them. The key outcomes of this work can be generalized to other waveguide structures, e.g. rectangular and dielectric waveguides, optical fibers, ect.., or to metasurface-bounded guiding structures, which give an easier path also to the design and realization of novel devices at microwave and optical frequencies based on the anomalous scattering of temporal metamaterials. 
(a)
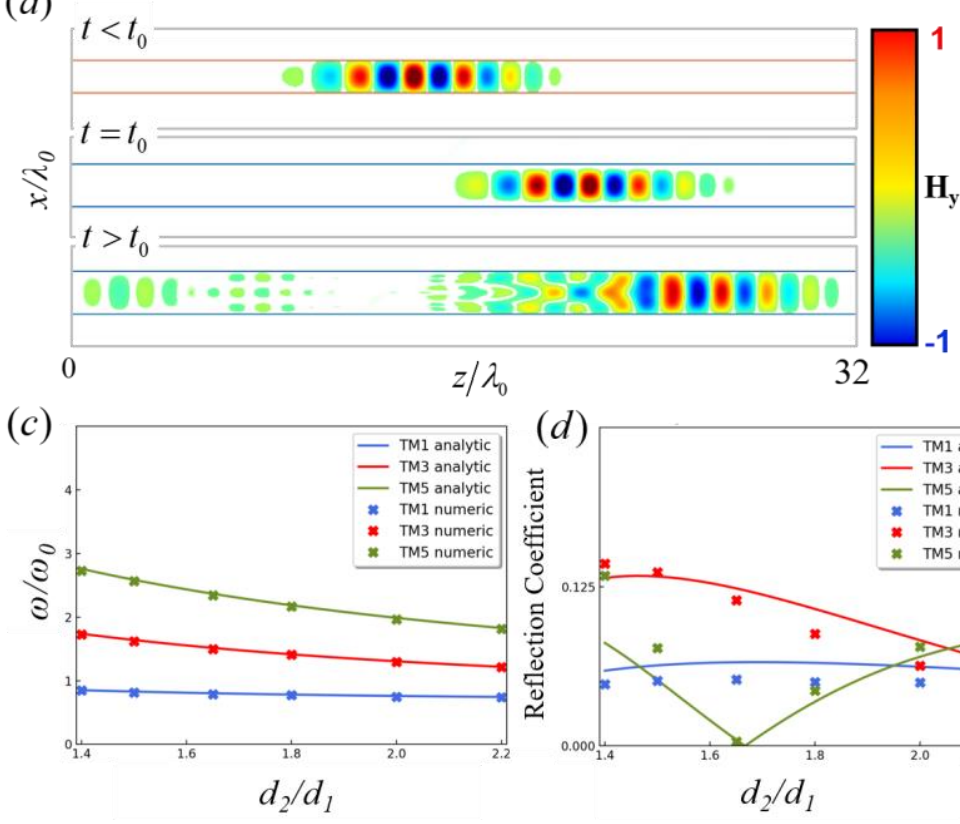

(b)
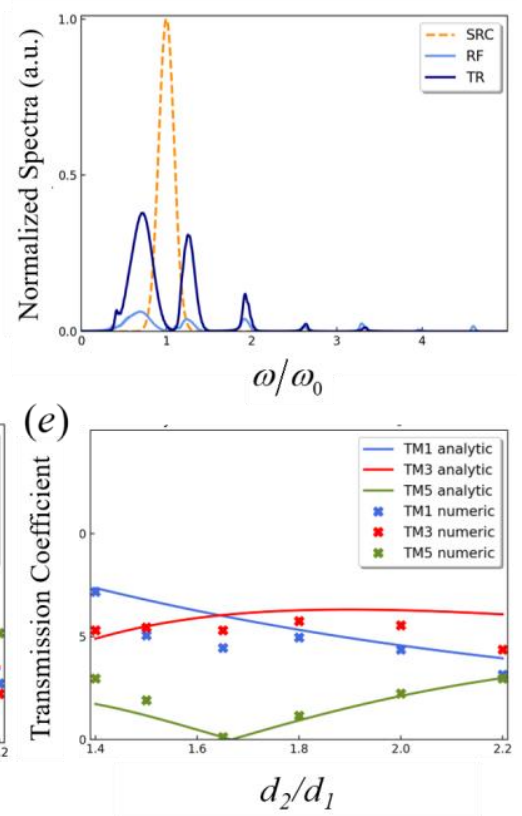

Fig. 3. (a) Snapshot in time of the H-field map within a PPWG with a boundary induced temporal interface at $t=t_{0}$; (b) Normalized spectra of the incident, reflected and transmitted field at a temporal interface induced by a changing of the waveguide dimensions from $1.0 d_{1}$ to $1.2 d_{1}$; (c)-(d) Comparison between analytical and numerical results of (c) frequencies, (d) reflection coefficient, and (e) transmission coefficient of the excited modes after the boundary induced temporal interface as a function of the ratio $d_{2} / d_{1}$ between the final and initial waveguide dimension.

\section{REFERENCES}

1. F. R. Morgenthaler, "Velocity Modulation of Electromagnetic Waves," IEEE Trans. Microw. Theory Tech. 6, 167-172 (1958).

2. Y. Xiao, D. N. Maywar, and G. P. Agrawal, "Reflection and transmission of electromagnetic waves at a temporal boundary," Opt. Lett. 39, 574-577 (2014).

3. C. Caloz and Z.-L. Deck-Léger, "Spacetime Metamaterials - Part I : General Concepts," IEEE Trans. Antennas Propag. 68, 1569-1582 (2020).

4. C. Caloz and Z.-L. Deck-Léger, "Spacetime Metamaterials-Part II: Theory and Applications," IEEE Trans. Antennas Propag. 68, 379-381 (2020). 
5. D. Ramaccia, A. Alù, A. Toscano, and F. Bilotti, "Temporal multilayer structures for designing higher-order transfer functions using time-varying metamaterials," Appl. Phys. Lett. 118, 101901 (2021).

6. A. Akbarzadeh, N. Chamanara, and C. Caloz, "Inverse prism based on temporal discontinuity and spatial dispersion," arXiv 2, 1-6 (2017).

7. V. Pacheco-Peña and N. Engheta, "Temporal aiming," Light Sci. Appl. 9, (2020).

8. V. Pacheco-Peña and N. Engheta, "Effective medium concept in temporal metamaterials," Nanophotonics 9, 379-391 (2020).

9. V. Pacheco-Peña and N. Engheta, "Anti-reflection temporal coatings," Optica 7, 323-331 (2020).

10. D. Ramaccia, A. Toscano, and F. Bilotti, "Light propagation through metamaterial temporal slabs: Reflection, refraction, and special cases," Opt. Lett. 45, (2020).

11. C. Caloz and Z. L. Deck-Léger, "Spacetime metamaterials," arXiv (2019).

12. H. Li and A. Alù, "Temporal switching to extend the bandwidth of thin absorbers," Optica 8, 24 (2021).

13. Z. Wu and A. Grbic, "Serrodyne Frequency Translation Using Time-Modulated Metasurfaces," IEEE Trans. Antennas Propag. 68, 1599-1606 (2020).

14. D. Ramaccia, D. L. Sounas, A. Alu, A. Toscano, and F. Bilotti, "Phase-Induced Frequency Conversion and Doppler Effect with Time-Modulated Metasurfaces," IEEE Trans. Antennas Propag. 68, 1607-1617 (2020).

15. B. Liu, H. Giddens, Y. Li, Y. He, S.-W. Wong, and Y. Hao, "Design and experimental demonstration of Doppler cloak from spatiotemporally modulated metamaterials based on rotational Doppler effect," Opt. Express 28, (2020).

16. L. Zhang, X. Q. Chen, S. Liu, Q. Zhang, J. Zhao, J. Y. Dai, G. D. Bai, X. Wan, Q. Cheng, G. Castaldi, V. Galdi, and T. J. Cui, "Space-time-coding digital metasurfaces," Nat. Commun. 9, 4334 (2018).

17. J. Zhao, X. Yang, J. Y. Dai, Q. Cheng, X. Li, N. H. Qi, J. C. Ke, G. D. Bai, S. Liu, S. Jin, A. Alù, and T. J. Cui, "Programmable time-domain digital-coding metasurface for non-linear harmonic manipulation and new wireless communication systems," Natl. Sci. Rev. 6, 231-238 (2019). 
18. X. Ma, M. S. Mirmoosa, and S. A. Tretyakov, "Parallel-Plate Waveguides Formed by Penetrable Metasurfaces," IEEE Trans. Antennas Propag. 68, 1773-1785 (2020).

19. R. E. Collin, Field Theory of Guided Waves, 2nd Edition (Wiley-IEEE Press, 1991).

20. D. M. Pozar, Microwave Engineering (Wiley, 2012).

21. J. C. Strikwerda, Finite Difference Schemes and Partial Differential Equations, Second Edition (Society for Industrial and Applied Mathematics, 2004). 